\title{
Reliability Analysis of the Control and Automation Systems in Electrical Substations
}

This paper was downloaded from TechRxiv (https://www.techrxiv.org).

LICENSE

CC BY 4.0

SUBMISSION DATE / POSTED DATE

$15-11-2021 / 29-11-2021$

\section{CITATION}

Zúñiga, Andrés; Branco, Paulo; Fernandes, João F. P.; Tarquinio, João P. S. (2021): Reliability Analysis of the Control and Automation Systems in Electrical Substations. TechRxiv. Preprint.

https://doi.org/10.36227/techrxiv.17014187.v1

$\mathrm{DOI}$

10.36227/techrxiv.17014187.v1 


\title{
Reliability Analysis of the Control and Automation Systems in Electrical Substations
}

\author{
João P. S. Tarquínio ${ }^{\mathrm{a}}$, Andrés A. Zúñiga ${ }^{\mathrm{b}^{*}}$, João F. P. Fernandes ${ }^{\mathrm{b}}$, Paulo J. Costa Branco ${ }^{\mathrm{b}}$ \\ ${ }^{a}$ Instituto Superior Técnico, Universidade de Lisboa, Av. Rovisco Pais 1, 1049-001 Lisboa, Portugal \\ ${ }^{b}$ IDMEC, Instituto Superior Técnico, Universidade de Lisboa, Av. Rovisco Pais 1, 1049-001 Lisboa, Portugal
}

\begin{abstract}
Demands for better electricity quality and the evolution of the electric power system are the most significant factors for the progress of substation automation systems. With continuous improvements in the fields of electronics and communication technologies, the electric power system has become even more complex. Moreover, interruptions in electricity supply can cause catastrophic events and there is an increasing effort for electricity to be continuously available on demand. Reliability analyses play a crucial role in power system planning, design, and operation. Therefore, many reliability assessment approaches have been proposed. However, assessing the impact of failures on secondary equipment in the power system can be extremely complex. This work consists in a detailed reliability analysis of the control, automation, and communication equipment in an IEC61850 based digital electrical substation considering four different communication architectures. Three preventive maintenance plans are defined according to Birnbaum's importance measure in order to evaluate if a more detailed planning proves to be beneficial. The method used to perform the substation's reliability and availability analysis consists in a Markov-Monte Carlo algorithm. Moreover, a quantitative cost analysis throughout the substation's life cycle is conducted to conclude about the economic feasibility of each architecture, allowing more objective decisions to be taken when considering the reliability requirements for electric power systems.
\end{abstract}

Keywords: Substation Automation Systems; Reliability; Markov Chain; Monte Carlo simulation; IEC61850

Abbreviation: SAS, Substation Automation Systems; IEC, International Electrotechnical Commission; IED, Intelligent Electronic Devices; NCC, Network Control Center; SCADA, Supervisory Control and Data Acquisition; LAN, Local Area Network; TTF, Time to Failure; MTTF, Mean Time to Failure; MTTR, Mean Time to Repair; MU, Merging Unit; SV, Server; HMI, Human-Mechine Interface; TS, Time Synchronization Unit; SW, Ethernet Switch; Ssw, Station Switch; EL, Ethernet Cable Link; FO, Fiber Optics Link.

\section{Introduction}

In electric power systems, substations are a crucial element in the transmission and distribution of electrical energy. These infrastructures are mainly responsible for transforming electrical energy by stepping-up or down the voltage depending on the power system's requirements, whether it is to deliver energy to the electric grid or to supply the costumers.

Nowadays, the smart grid vision has increased due to policy and regulatory initiatives. The automation functions for monitoring, protection and control within a substation and recent improvements in the fields of electronics and communication technologies are provided by Substation Automation Systems (SAS).

The application of the International Electrotechnical Commission standard IEC 61850 in SAS brings significant changes to its instrumentation, monitoring, communication, control and protection systems, providing more flexibility and a better performance of the SAS architectures [1].

The electricity supply system represents an extremely complex and highly integrated system, where the failure of one single element can cause interruptions. The effects of these interruptions range from inconveniencing a small number of residents closer to the failure origin, to major and widespread disruptions of supply, which can cause catastrophic events [2]. Due to this failure scenario, many reliability assessment approaches have been proposed; however, most of these are focused exclusively on either the primary equipment or the secondary equipment separately. In addition, assessing the impact of failures on secondary equipment in the power system is extremely complex.

The underlying motivation of this dissertation is to study the existing technologies developed for the automation of substations, provided that these will enable a more reliable and efficient monitoring, operation, control and protection, as well as an increase in operator safety and quality of service.

The purpose of this work is to assess the reliability in electric substations considering the new technologies and equipment associated to control, automation and communication based on the IEC 61850 standard. The impact of

\footnotetext{
* Corresponding author. Tel.: +351-218417170; fax: +351-218417190.

E-mail address: andres.zuniga@tecnico.ulisboa.pt
} 
failure of SAS equipment in the operation of the substation is evaluated through a detailed analysis, which encompasses the reliability and availability of the substation and an economic evaluation of the costs carried throughout its lifetime. This is performed considering four different communication architectures, responsible for the connection of all equipment in study. The goal of this work is to conclude about which architecture guarantees the best reliability and implies the lowest possible expenses.

\section{Background}

\subsection{Digital Substations}

Digital substation is a term used to refer to substations which is managed by control and communications systems based on the IEC 61850 standard. Over the years, as computing power became greater and cheaper, fewer devices were required to carry out the same functions. Due to the integration of several functionalities and communications within the devices, the traditionally separated protection, monitoring, and control functions converged, forming the currently known Substation Automation Systems [3].

Modern Intelligent Electronic Devices IEDs are numerical protection relays, which encompass, in addition to protection functions, a variety of features related with automation, control, monitoring, supervision, among others.

Despite the advantages brought by IEDs to power system's protection, automation, and control, the introduction of these devices replaces some of the issues of previous generations of relays with new ones, such as software version control, data management, testing, and commissioning [4]. These issues must be continuously addressed both from manufacturers and costumers to ensure that today's smart grid requirements are met.

\subsection{IEC 61850 Standard}

With the evolution of the secondary equipment, new problems arise due to the fact that there is a variety of manufacturers developing their own devices, which communicate in a wide spectrum of protocols. This resulted in an extremely complex problem for engineers, since many of the protocols used are not interoperable, meaning that the devices are not meant to communicate with each other natively. Furthermore, with the increasing integration of renewable energies in the electric power grid, which introduce a different set of manufacturers, protocols, and electricity capable of disturbing the stability of grid-supplied electricity (e.g., frequency of power supplied), a need for a common standard is of the utmost importance [5].

The IEC 61850 standard was designed by leading SAS experts throughout the world to simplify the process of automation within electric substations. This standard defines how data is executed and stored, and it also covers device specifications and provides equipment interoperability, interchangeability, and redundancy. Other major benefits associated to the implementation of the IEC 61850 standard are related to free configuration, simpler architecture, and resource's savings [6].

A typical architecture of the IEC 61850 based SAS is shown in Fig. 1.

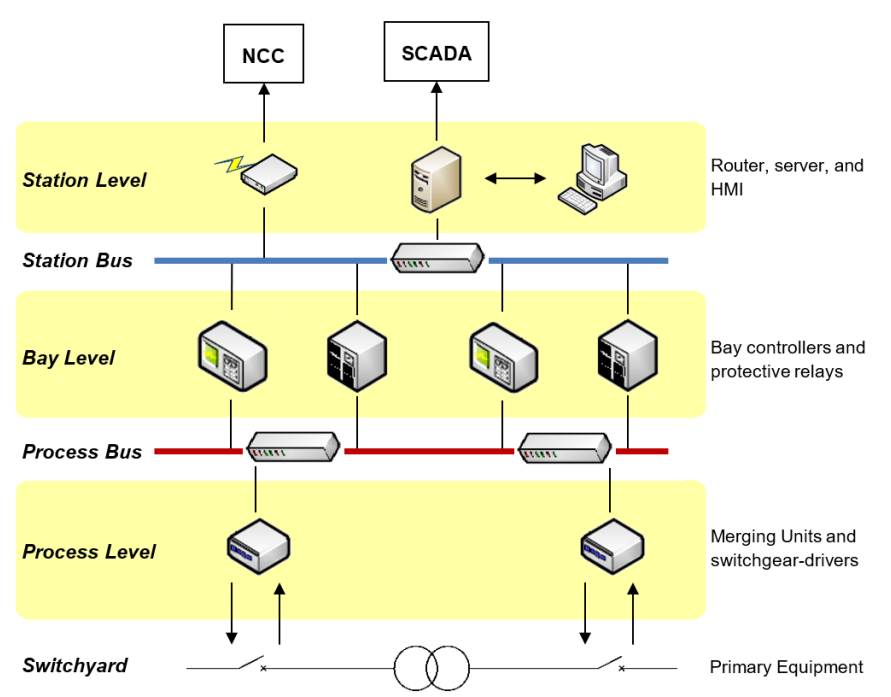

Fig. 1. Architecture of an IEC 61850 Substation Automation System [7].

This architecture consists of three levels (process level, bay level, and station level) and two buses (process bus and station bus). The current and voltage values in the substation are treated in the process level, mainly for protection purposes, by merging units. Protection and control IEDs are included in the bay level. The station level 
includes a gateway which enables remote access to the Network Control Center (NCC), a station server which provides the human-machine interface functionality and communication to the SCADA system [3]. Regarding the process and station buses, these are responsible for the complete information exchange between levels using network switches, which are required to network multiple devices in a LAN.

\section{Fundamental Concepts}

The failures in the systems generally occur in an aleatory way; however, the failure state can be induced by suddenly variations in the normal operating conditions. Let $T$ be a random variable that represents the operating time of a system until a failure occurs, known as the Time to Failure TTF. Time to failure can be modelled using a probability density function $f(t)$. The cumulative distribution function $F(t)$ is the probability of a failure occur in the time interval $(0, t]$ and it is known as probability of failure. The reliability, denoted by $R(t)$, is defined as the probability that no failure will occurs during a stated time interval $(0, t]$, as shown in equation (1) [8].

$$
R(t)=1-F(t)=1-\int_{0}^{t} f(u) d u=\int_{t}^{+\infty} f(u) d u
$$

The system's failure rate, denoted by $\lambda(t)$, is defined as the rate of failure of a component or system, given that the failure has not occurred prior to time $\mathrm{t}$, and can also be expressed as the ratio of probability density function $f(t)$ over reliability function $R(t)$, as shown in, as proven in equation (2) [8].

$$
\lambda(t)=\lim _{\Delta t \rightarrow 0} \frac{F(t+\Delta t)-F(t)}{\Delta t} \frac{1}{R(t)}=\frac{f(t)}{R(t)}
$$

A rough approximation of the behavior of the failure rate function with time regarding components and systems is given in Fig. 2, which is known as the bathtub curve due to its characteristic shape.

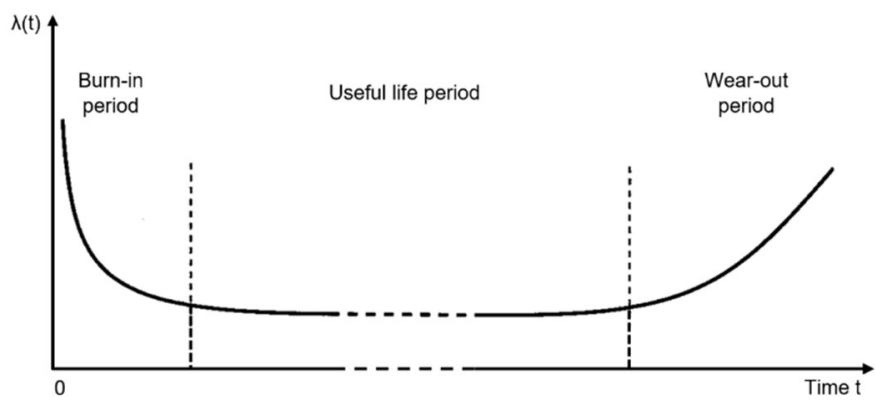

Fig. 2. Bathtub curve of an electrical component or system, adapted from [8].

Alternatively, it is also possible to compute the time between failures of a component. Therefore, the mean time to failure $(M T T F)$ is the expected time that a component takes to fail. In the case that the component is repairable, it is also necessary to take into account the concept of mean time to repair (MTTR). This corresponds to the expected time that a component takes to be repaired and it is the inverse of the repair rate, $\mu$.

Since reliability work related with electrical components usually deals with the useful life period of the bathtub, in this work it is assumed the constant failure rate model. This model is defined by a constant failure rate $\lambda$ and the time to failure is represented by an exponential probability distribution. Reliability can then be defined as in equation (3) [8].

$$
R(t)=e^{-\lambda t}
$$

Finally, the instantaneous availability of a component, $A(t)$, is its ability to be kept in a functioning state at specific time $t$. The long run average availability, $A_{\text {avg }}$, is the proportion of time that the system is operating and is expressed in terms in terms of $\lambda$ and $\mu$, by equation (4) [8].

$$
A_{\text {avg }}=\frac{M T T F}{M T T F+M T T R}=\frac{\mu}{\mu+\lambda}
$$

Depending on the complexity of a system and the number of different components integrated in it, it may be beneficial to evaluate the importance of each component to the overall system.

The Birnbaum's component importance measure gives the rate of change of the system's reliability accordingly to changes in the reliability of component $i$ [8]. The importance of component $i$ to the system at time $t$, $I^{B}(i \mid t)$, is given by the partial derivative of the system's reliability, $R_{s y s}(t)$, with respect to the reliability of the component, $R_{i}(t)$, as shown in equation (5) [8]. 


$$
I^{B}(i \mid t)=\frac{\partial R_{s y s}(t)}{\partial R_{i}(t)}
$$

Moreover, Birnbaum's critical importance, $I^{C R}(i \mid t)$, measures the probability that a specific component $i$ is the cause of a total system failure after a time $t$. Analytically, the critical importance is defined by equation (6) [8].

$$
I^{C R}(i \mid t)=I^{B}(i \mid t) \frac{1-R_{i}(t)}{1-R_{s y s}(t)}
$$

\subsection{Markov Process}

In the field of reliability assessment, Markov models, which are stochastic processes described by a set of all possible states of the system and the transitions between these states, are commonly used. The state transitions are usually given by the failure rates, $\lambda$, and the repair rates, $\mu$. Moreover, the collection of all possible states, represented by $S$, is called the state space. For each state, there is a probability associated to it, $P_{i}(t)$, which is the probability of the system being in state $i$ at time $t$ [8].

In order to compute the state probabilities, Markov processes can be solved by means of differential equations. These can be represented in matrix form as shown in equation (7), where $\mathbf{M}$ is the Markov matrix, also known as transition matrix.

$$
\frac{d P(t)}{d t}=\mathbf{M} \cdot P(t)
$$

Then, to compute the reliability of the system, and since the sum of all state probabilities is equal to one, the reliability can be simply obtained by subtracting the sum of the probabilities corresponding to failed states, $P_{f}(t)$, to one, as shown in equation (8).

$$
R(t)=1-\sum P_{f}(t)
$$

\subsection{Monte Carlo Simulation}

The Monte Carlo method is the general designation for stochastic simulation using random variates. The idea behind this method is the development of an equivalent stochastic process that behaves as much as possible as the real system under analysis. Thereby, a large number of simulations are required in order to cover as much operational scenarios as possible [2].

Since the constant failure rate model is considered in this work, in which the time to failure is represented by an exponential probability distribution, exponentially distributed random variables can be generated through the inverse transform method. This method allows to use a random number generator that samples from a uniform distribution between $[0,1]$ to sample from another distribution.

The randomness of the Monte Carlo simulation is then applied to its most variable factors, which correspond to the failure and repair times of the components. Additionally, these factors affect mostly the availability of systems, this being the main reason for choosing Monte Carlo simulation for its calculation.

The Monte Carlo procedure adopted in this work can be described by the following steps:

1. The period of simulation is divided into an equal number of time periods called trials;

2. At each trial, a set of random numbers are generated and confronted against the corresponding probability of component failure or repair during the trial period;

- If the component is in operation at the start of the trial and the generated random number is higher than its failure probability, the component will be on a failed state at the end of the trial;

- If the component was on a failed state at the start of the trial and the generated random number is higher than its repair probability, the component will be in operation at the end of the trial;

3. The state of the system is determined at each trial depending on its component's states and logical connections;

4. The total operational time of the system is computed, and the simulation is then repeated $\mathrm{n}$ times in order to obtain the average availability of the system, as given in equation (9), where $A_{i}$ is the is the availability obtained in the $\mathrm{i}^{\text {th }}$ repetition of the Monte Carlo procedure, $n_{s i m}$ corresponds to the number of simulations performed, Top is the total operational time and Tsim is the total simulation time.

$$
A_{\text {avg }}=\sum_{i=1}^{n} \frac{A_{i}}{n_{\text {sim }}}=\sum_{i=1}^{n} \frac{T_{o p}}{T \operatorname{sim}_{i}}
$$




\section{Case study}

The proposed approach was tested on a real $30 \mathrm{MW}$ combined cycle power plant installed in Luanda, Angola. The power plant's substation actually has a SCADA-based automation and control system. For confidentiality reasons, no further data about the system is given.

The substation is connected to the electrical power system through a $60 \mathrm{kV}$ power line, the power plant's generators are connected at $11 \mathrm{kV}$ voltage level and the loads and auxiliary circuits are powered at $15 \mathrm{kV}$. The simplified substation model is composed of one gas turbine (G1), one steam turbine (G2), 5 busbars (B1-B5), 2 step-down transformers (T1 and T2), 2 step-up transformers (T3 and T4), 4 equivalent loads (L1 - L4), and 16 circuit breakers (CB1 - CB16), as shown in Fig. 3.

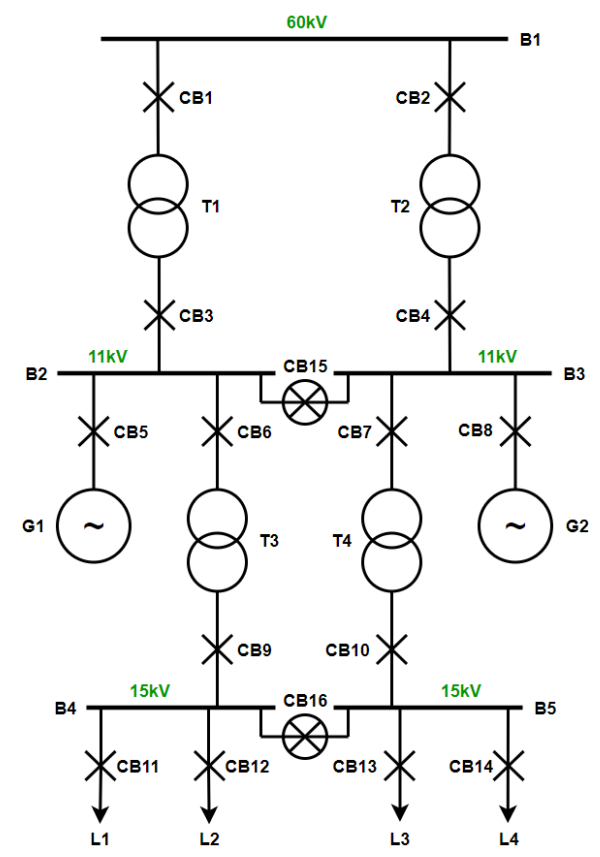

Fig. 3. Single-line diagram of the case study substation [7].

To model this substation as a smart substation, it was considered all equipment to model the substation's Substation Automation Systems as an IEC 61850-based SAS, according to typical architecture shown in Fig. 1.

For the process level are considered the Merging Units (MUs), responsible for digitizing and sending the current and voltage values to the bay level devices. In previous versions of SAS architectures, IEDs would send trip commands via the process bus to breaker IEDs, which then trip the circuit breakers; however, recent models of MUs already include an actuator control order function, meaning that there is no need for a breaker IEDs in newest versions of SAS architectures. In the bay level, only protection and control IEDs are considered. In the station level are considered a Server (SV), a Human-Machine Interface (HMI) and Time Synchronization Units (TS).

To guarantee communication between levels, two different types of ethernet switches are considered: one type installed the process bus, named as Ethernet Switches (SW), and another installed in the station bus, named as Station Switches (Ssw). Ethernet Cable Links (EL) are considered as link between the server and the HMI, whereas, for the remaining devices Fiber Optic Links (FO) are considered.

\subsection{Proposed communication architectures}

An important aspect to consider in the IEC 61850-based SAS reliability is the communication architecture. This work considers four different SAS communication architectures as proposed in [9].

The cascading architecture, shown in Fig. 4, where the ethernet switches from the process bus are connected in chain with an open loop configuration and only one of these is connected directly to the station switch. Its main advantage is the low cost because the shorter wiring, and presents no redundancy and latency as the main disadvantage. 


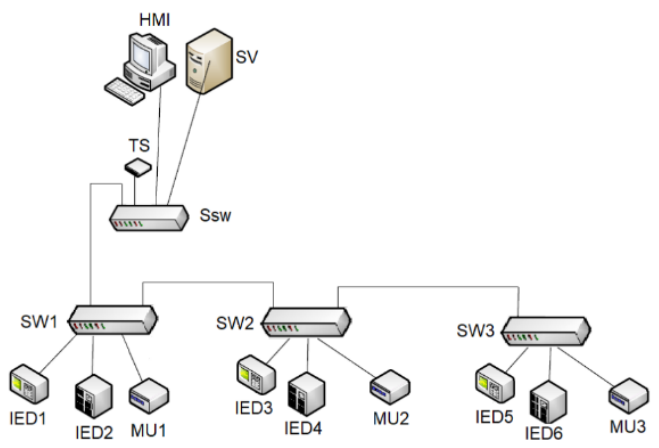

Fig. 4. Cascaded architecture, adapted from [9].

The ring architecture (Fig. 5) is very similar to the cascaded architecture, except that the loop is closed, with both the first switch (SW1) and the last switch (SW3) connected directly to the station switch Ssw1. This architecture has de advantage of redundancy, it is cost effective in wiring terms (similar to cascading) and allows the use of IEEE 802.1w Rapid Spanning Tree Protocol for sub-second reconfiguration. As disadvantages we can identify the latency, and the fact that all the switches considered in this architecture must be managed switches.

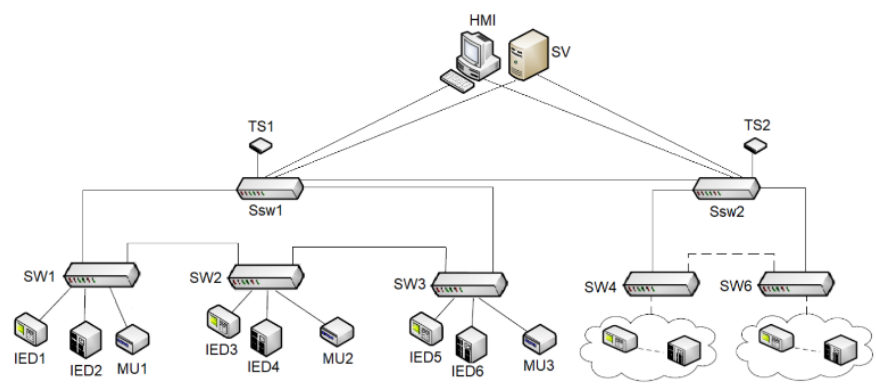

Fig. 5. Ring architecture, adapted from [9].

In a star architecture (Fig. 6), each ethernet switch has a direct connection to all station switches in order to form a star configuration, which in turn are connected all together. Its main advantages is the lowest latency but has de disadvantage of no redundancy.

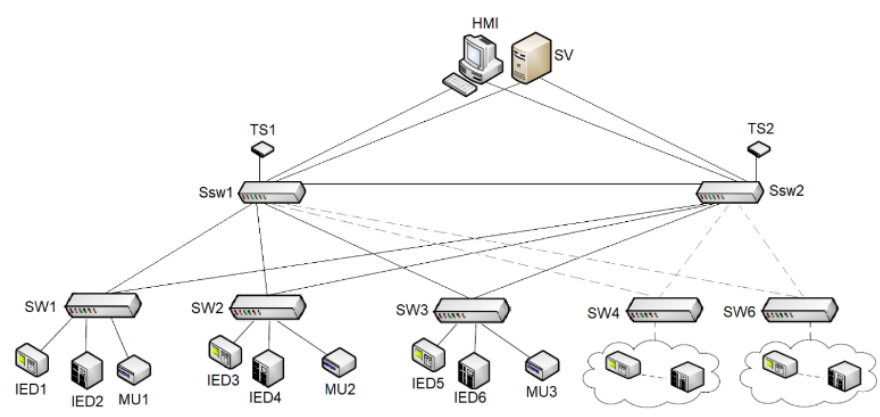

Fig. 6. Star architecture, adapted from [9].

The last architecture assumed in this work corresponds to the fault tolerant redundant ring architecture, which provides two completely redundant rings of ethernet switches in the process bus, as shown in Fig. 7.

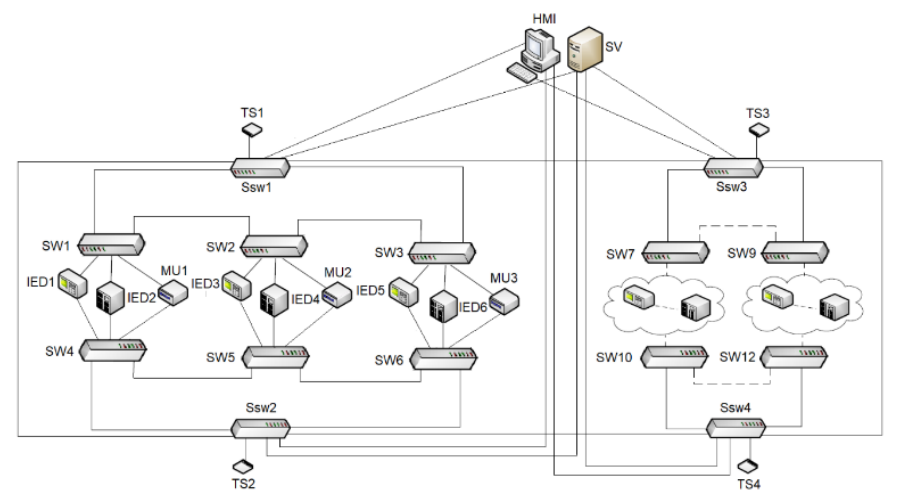

Fig. 7. Redundant ring architecture, adapted from [9]. 
It is expected that if redundancy increases, the reliability and the availability also increase; nevertheless, the results for the economical evaluation are not so predictable. Although the increasing redundancy of the architectures reduces the costs of penalties it requires a greater investment in preventive maintenance.

\subsection{Reliability Data}

The reliability data was extracted from academic references and databases; Table 1 shows the failure rate and MTTR considered for the SAS equipment.

Table 1. Failure and MTTR of each SAS component.

\begin{tabular}{lcccc}
\hline \multicolumn{1}{c}{ SAS component } & Abbreviation & $\begin{array}{c}1 \\
\text { (f/year) }\end{array}$ & $\begin{array}{c}\text { MTTR } \\
\text { (hours) }\end{array}$ & Source \\
\hline $\begin{array}{l}\text { Merging Unit } \\
\text { Intelligent Electronic }\end{array}$ & MU & 0.0067 & 24 & {$[10]$} \\
$\begin{array}{l}\text { Device } \\
\text { Server }\end{array}$ & IED & 0.0526 & 24 & {$[11]$} \\
$\begin{array}{l}\text { Human Machine } \\
\text { Interface }\end{array}$ & HMI & 0.0270 & 24 & {$[12]$} \\
Time Synchronization & TS & 0.1720 & 24 & {$[13]$} \\
Unit & & 0.0067 & 24 & {$[10]$} \\
Ethernet Switch & SW & 0.0225 & 24 & {$[14]$} \\
Station Switch & Ssw & 0.0225 & 24 & {$[14]$} \\
Ethernet Link & EL & 0.0010 & 24 & {$[11]$} \\
Fiber optic Link & FO & 0.0438 & 24 & {$[15]$} \\
\hline
\end{tabular}

\subsection{Reliability Analysis Procedure}

In this work it is assumed that failures in SAS equipment will only affect circuit breaker operation, as it is the only primary equipment whose operation depends on the SAS components. It is considered that circuit breakers can be affected by two failure modes caused due to SAS failures:

- Miss operation - Unintended CB operation;

- Fail to operate - CB did not operate when intended.

In order to establish a relation between failures of SAS equipment and substation's inoperability, it is necessary to identify all possible failure modes that affect SAS components. A previous work in our research team identified the causes for each one of the SAS's failure modes that can be a possible trigger for one of the previously mentioned circuit breaker faults [11].

We considered the implementation of at least one ethernet switch per busbar and inter-busbars (where CB15 and CB16 are located) for all communication architectures in study, and we assumed that station switches are connected to no more than three ethernet switches. For the sake of simplicity, it is also considered that circuit breakers fail exclusively if both its corresponding protection and control IEDs fail, triggering the same circuit breaker failure mode. Regarding time synchronization, it is assumed that one unit is connected to each station switch to synchronize all merging units.

\subsection{Reliability Model}

Once established the relationship between failures in SAS equipment and the operation of the substation, the following step is to define the Markov chain of the substation to compute its reliability. For this purpose, four operational states corresponding to the number of loads supplied by the substation are considered. The Markov chain of the substation can be observed in Fig. 8 and the description of its states is as follows:

- $S 1$ - all loads supplied (operational state);

- $S 2$ - any three loads supplied (failed state);

- $S 3$ - any two loads supplied (failed state);

- $S 4$ - no loads supplied (failed state). 


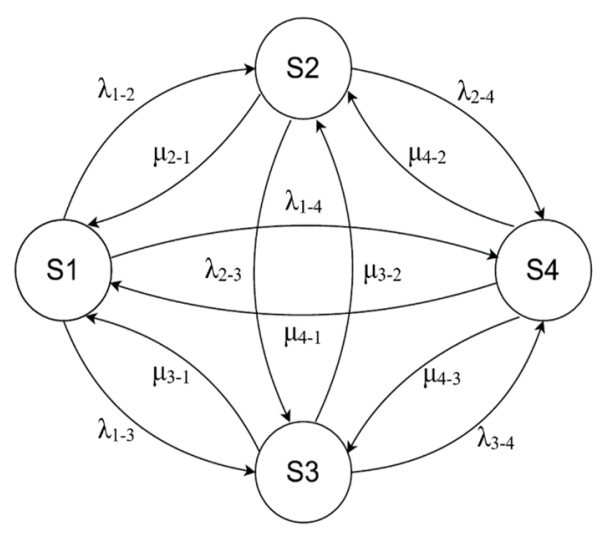

Fig. 8. Markov chain of the substation [7].

To compute the transition rates between states, RBDs were developed representing the possible combinations of circuit breakers' failure modes that, upon failure, cause the substation to interrupt the power supply.

Finally, the Monte Carlo simulation method is used to simulate events happening throughout the lifetime of the substation and, hence, computing its availability. In this work, it is assumed a substation's lifetime of 100 years, in order to have a broader spectrum of its operation with the purpose of studying its behavior even after the normal lifetime of substations, which is between 40 and 50 years.

In substations, the mindset for microprocessor-based equipment is that it should have a lifetime of 10 years. However, it is unlikely that this replacement cycle is fulfilled. Moreover, substations are sometimes expanded and not very often completely replaced. For that reason, a more accurate replacement cycle for this equipment is 15 or even 20 years [16].

\subsection{Preventive Maintenance Plans}

We define three different scenarios for preventive maintenance plans to be applied for each of the communication architectures, named as Plan 1, Plan 2 and Plan 3. Standard values of SAS equipment's maintenance periods available in the literature and consulted to professional engineers from the EREDES (Portugal) were used to define preventive maintenance plans; according to [17], maintenance periods for the components in study are situated between one and 12 years, with an average of 3.5 years.

For the first plan (Plan 1), we proposed a single maintenance period of 5 years for all components regardless their importance.

The Birbaum's critical importance $I^{C R}(i \mid t)$ computed for each componente after 20 years was used to define Plan 2 and Plan 3. Plan 2 was design considering two different maintenance frequencies: the most important components according to Birnbaum's importance measure will be maintained every 4 years and the remaining components every 6 years.

Plan 3 considers three different maintenance frequencies: the most critical components will be maintained every 3 years, the least critical important components are maintained each 7 years, and components with intermediate criticality importance are maintained every 5 years. It is assumed that SAS components are stochastically independent.

The maintenance plans for the cascaded and redundant ring communication architectures are shown in Table 2 and Table 3.

Table 2. Maintenance plans defined for cascading architecture [7].

\begin{tabular}{lccc} 
& \multicolumn{3}{c}{ Maintenance period (years) } \\
\hline \multicolumn{1}{c}{ SAS component } & Plan 1 & Plan 2 & Plan 3 \\
\hline Merging Unit (MU) & 5 & 6 & 5 \\
Intelligent Electronic Device (IED) & 5 & 6 & 5 \\
Server (SV) & 5 & 4 & 3 \\
Human Machine Interface (HMI) & 5 & 4 & 3 \\
Time Synchronization Unit (TS) & 5 & 6 & 5 \\
Ethernet Switch (SW) & 5 & 4 & 3 \\
Station Switch (Ssw) & 5 & 6 & 5 \\
Ethernet Link (EL) & 5 & 6 & 7
\end{tabular}


Table 3. Maintenance plans defined for redundant ring architecture [7].

\begin{tabular}{lccc} 
& \multicolumn{3}{c}{ Maintenance period (years) } \\
\hline \multicolumn{1}{c}{ SAS component } & Plan 1 & Plan 2 & Plan 3 \\
\hline Merging Unit (MU) & 5 & 6 & 5 \\
Intelligent Electronic Device (IED) & 5 & 6 & 5 \\
Server (SV) & 5 & 4 & 3 \\
Human Machine Interface (HMI) & 5 & 4 & 3 \\
Time Synchronization Unit (TS) & 5 & 6 & 5 \\
Ethernet Switch (SW) & 5 & 6 & 7 \\
Station Switch (Ssw) & 5 & 4 & 5 \\
Ethernet Link (EL) & 5 & 6 & 7 \\
Fiber optic Link (FO) & 5 & 6 & 5 \\
\hline
\end{tabular}

Since constant failure rate model is considered in this work, the effect of preventive maintenance must be applied directly in the reliability model of each component. The execution of preventive maintenance can increase the reliability of components, but the possibility of failure still exists. A preventive maintenance level, denoted by $\delta_{t}$, is considered to model the component's reliability improvement after the preventive maintenance was performed. The value of $\delta_{t}$ at each maintenance period can be computed according to equation (10).

$$
\delta_{t}=R_{0}(t-1)-R_{0}(t)
$$

The preventive maintenance level is added to the reliability value of the component in the previous year in order for the reliability in the maintenance time instant, $R\left(t_{m}\right)$, to increase. The result of the application of this method into the reliability of components should be as represented in Fig. 9.

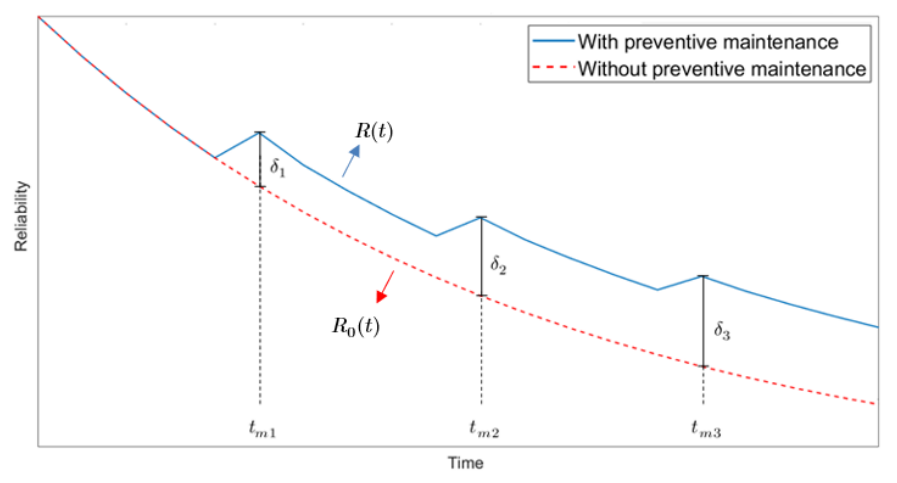

Fig. 9. Effect of preventive maintenance in the reliability of the components [7].

\subsection{Economic Evaluation Considerations}

We performed a detailed study of the costs underlying the operation of the substation for the four communication architectures. In order to obtain the amount of money spent during substation's lifecycle, referred to the present time, for the computation of discounted costs it is assumed a discount rate of $7 \%$. Furthermore, investment, maintenance, and unavailability costs are considered for the economic evaluation.

The investment consists of the sum of the average price of each SAS component according to the Portuguese market and its implementation in the substation. For the communication links, it is considered that ethernet and fiber optic connections have an average length of 10 and 20 meter, respectively. Table 4 shows the investement costs considered in the simulations.

Table 4. Investment costs for the communication architectures [7].

$\begin{array}{ccccccccccc} & \text { MU } & \text { IED } & \text { SV } & \text { HMI } & \text { TS } & \text { SW } & \text { Ssw } & \text { EL } & \text { FO } & \text { Investment } \\ \text { (k€) } \\ \begin{array}{l}\text { Average } \\ \text { Price (€) }\end{array} & 3500.00 & 3537.50 & 2400.00 & 4300.00 & 7000.00 & 2600.00 & 3700.00 & 1.20 / \mathrm{m} & 2.00 / \mathrm{m} & \text { ( }\end{array}$




\begin{tabular}{|c|c|c|c|c|c|c|c|c|c|c|}
\hline \multicolumn{11}{|c|}{ Number of units/Length of the cables } \\
\hline Cascaded & 16 & 32 & 1 & 1 & 2 & 6 & 2 & $40 \mathrm{~m}$ & $1120 \mathrm{~m}$ & 215.19 \\
\hline Ring & 16 & 32 & 1 & 1 & 2 & 6 & 2 & $40 \mathrm{~m}$ & $1180 \mathrm{~m}$ & 215.30 \\
\hline Star & 16 & 32 & 1 & 1 & 2 & 6 & 2 & $40 \mathrm{~m}$ & $1240 \mathrm{~m}$ & 215.43 \\
\hline $\begin{array}{c}\text { Redundant } \\
\text { Ring }\end{array}$ & 16 & 32 & 1 & 1 & 4 & 12 & 4 & $80 \mathrm{~m}$ & $2400 \mathrm{~m}$ & 254.80 \\
\hline
\end{tabular}

Regarding maintenance costs, both preventive and corrective maintenance are considered. Considering that the case study substation is located in Portugal, transportation costs are computed considering an average round trip of four hours (two hours per trip). In addition, gas and tool expenses are assumed to be of $40 €$ and $20 €$ per round trip, respectively. Since maintenance requires the intervention of specialized operators, the necessity of three operators with an average cost of $50 €$ per hour, working 8 hours per day is considered. It is also considered that each operator executes the required preventive maintenance procedures in an average of time of 30 minutes per component and 5 minutes per communication link. As for corrective maintenance procedures, besides transportation and operational costs, it is considered a repair cost of a half of the component's average price.

The unavailability costs consist of the penalties for energy not supplied to the costumers. In this work, it is assumed that these expenses correspond to an estimate of the income which would have been received if there was no interruption in power supply. Thus, the unavailability costs, $C_{u n v}$, are computed according to equation (11), where one considers the price of electricity in Portugal, $C_{\text {elec }}$, the profit margin from this value that comes from the commercialization of energy, $m$, the power delivered by the substation to each one of the loads, $P_{\text {Load }}$, the number of loads which are not being supplied, $n_{\text {down }}$, and the down time, $t_{\text {down }}$, in hours.

$$
C_{\text {unv }}=C_{\text {elec }} \cdot m \cdot P_{\text {LOAD }} \cdot n_{\text {down }} \cdot t_{\text {down }}
$$

It is considered a value of $0.2154 € / \mathrm{kWh}$ for the price of electricity in Portugal and $38 \%$ for the profit margin. Furthermore, since loads and auxiliary circuits are powered at $15 \mathrm{kV}$ and assuming that the current demanded from each load is $1250 \mathrm{~A}$, the value of $P_{\text {Load }}$ is $18.75 \mathrm{MW}$. The remaining variables are dependent on the Monte Carlo simulation.

\section{Result Analysis}

\subsection{Reliability, Availability and Total Costs}

For better clarity in the presentation and understanding of the results, these are presented according to the following order:

- Reliability;

- Availability;

- Total Discounted Costs.

Then, for the sake of simplicity, only the results obtained for the least and most redundant architectures (cascaded and redundant ring) are presented.

Starting with the reliability analysis of the substation, the reliability curves for each substation can be observed in Fig. 10 and Fig. 11, where only 40 years are considered, as the reliability behavior repeats every time SAS components are replaced. 


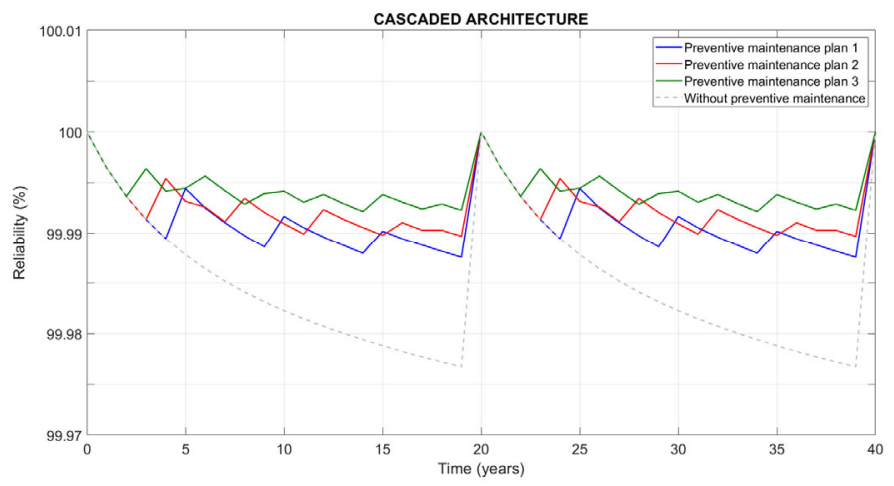

Fig. 10. Reliability curves for the cascade architecture [7].

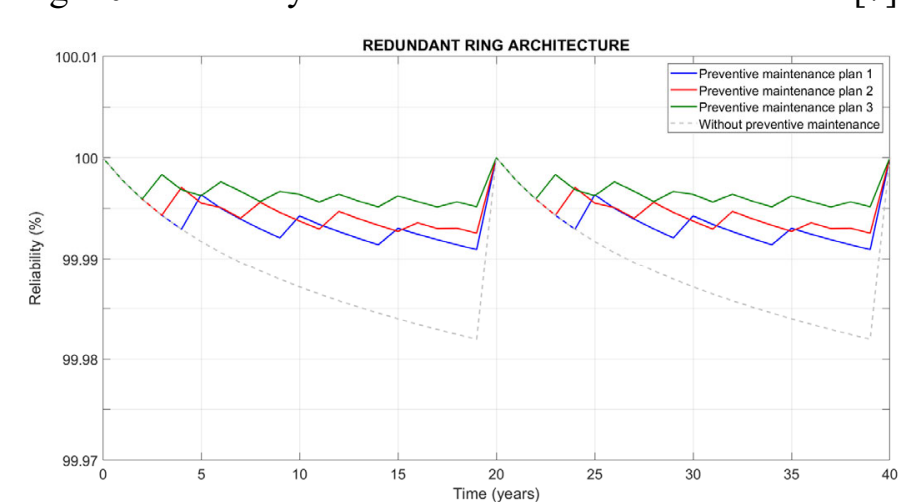

Fig. 11. Reliability curves for the redundant ring architecture [7].

The most obvious observation is the high reliability value. This is an expected result, since this work focuses on the study of the impact of failures of electronic equipment in substations, which are characterized by considerably low failure rate values. In addition, there is an improvement in reliability with the implementation of the defined maintenance plans. In turn, reliability becomes increasingly higher as the maintenance plan becomes more detailed. In the same sense, the behavior of its curve tends to converge in a straight line, resulting from the increasing number of maintenance periods in each plan. It is also possible to see that reliability is significantly higher considering the redundant ring architecture than in the cascaded architecture.

The analysis of the availability of the substation follows. Considering that this was calculated from the Monte Carlo simulation, it is possible to determine its mean value and variance over the lifetime considered for the substation. Thus, the average availability value is represented by a curve and its variance by the surrounding-colored area, as it can be seen in Fig. 12 and Fig. 13.

Similar to reliability, and as expected, the average availability of the substation presents a high value as well, regardless the maintenance plans and architectures considered. It is worth highlighting the significant difference between the average availability obtained in the scenario without maintenance and in the others. Furthermore, it is verified that the average availability increases with each maintenance plan. However, it drops visibly at the end of a 20-year cycle, which coincides with the period in which all SAS equipment is replaced. Contrariwise, the variance increases since there is greater uncertainty in the behavior of the components in the substation over time. There is also a more uniform distribution of variance over time between the various maintenance plans, where the peaks do not stand out so much in relation to the beginning of a new 20 -year cycle. This behavior is similar to that of substation reliability, where the difference between the maximum and minimum value becomes smaller at each plan.

Regarding communication architectures, and similarly to what happens with reliability, the average availability of the substation is directly related to the increase in redundancy guaranteed by each architecture. As for variance, its behavior does not vary depending on the chosen architecture. 
Fig. 12. Availability of the substation for the cascaded architecture [7].
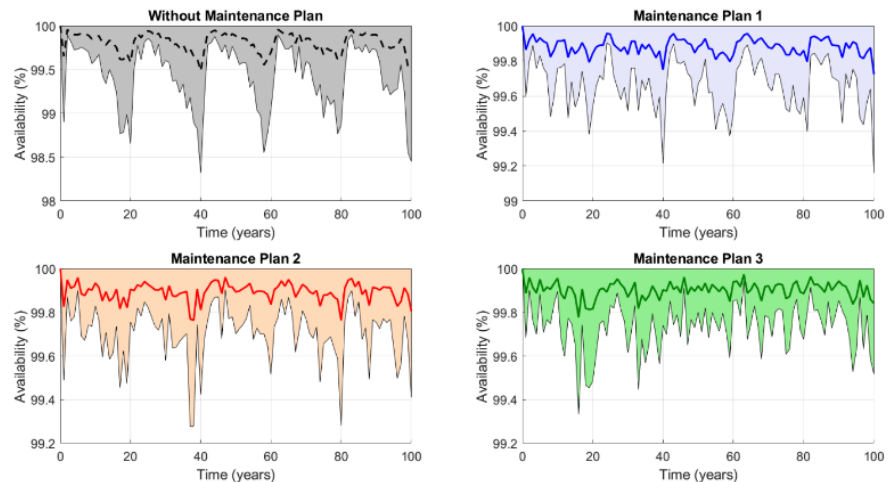

REDUNDANT RING ARCHITECTURE
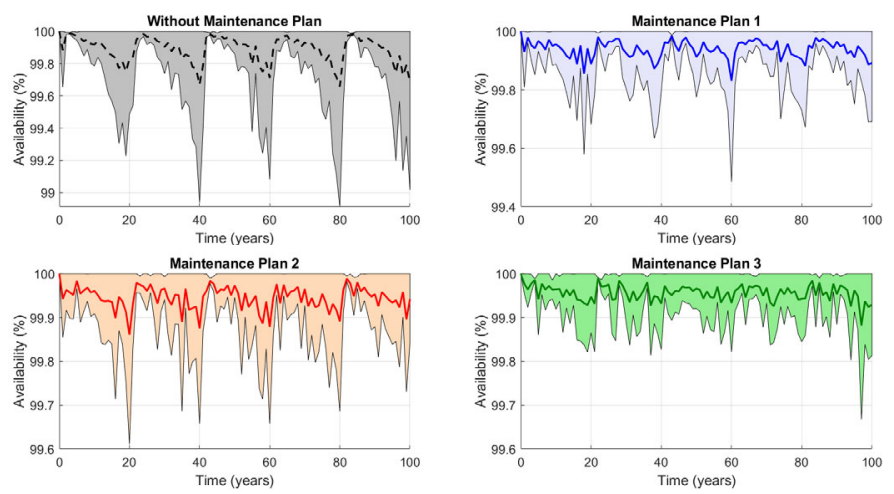

Fig. 13. Availability of the substation for the redundant ring architecture [7].

In order to understand if the advantages of a more detailed maintenance planning are extended to an economic perspective, similar graphs as the ones developed for the availability of the substation are presented for the total discounted costs, as shown in Fig. 14 and Fig. 15. The variance of this value over time was also taken into account, in order to understand if the variance in availability could be reflected in a wrong interpretation of the results obtained.

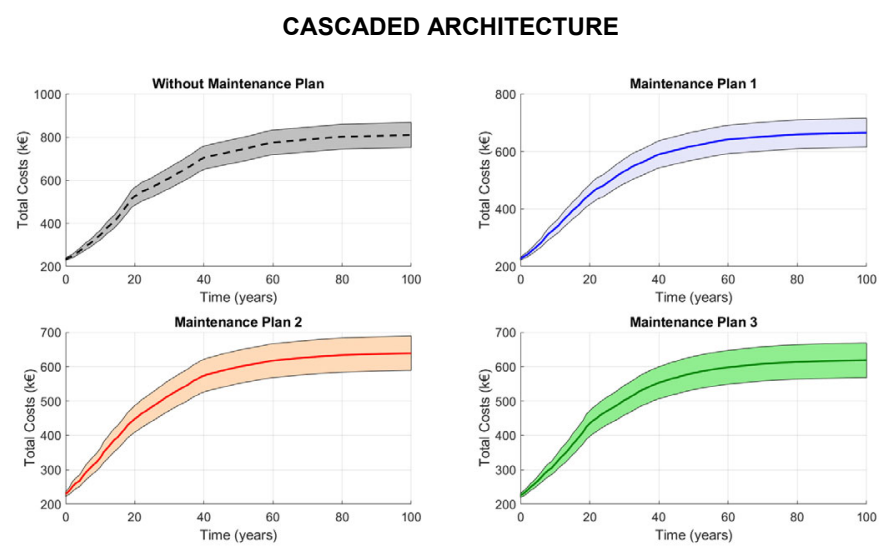

Fig. 14. Total discounted costs of the substation for the cascaded architecture [7]. 


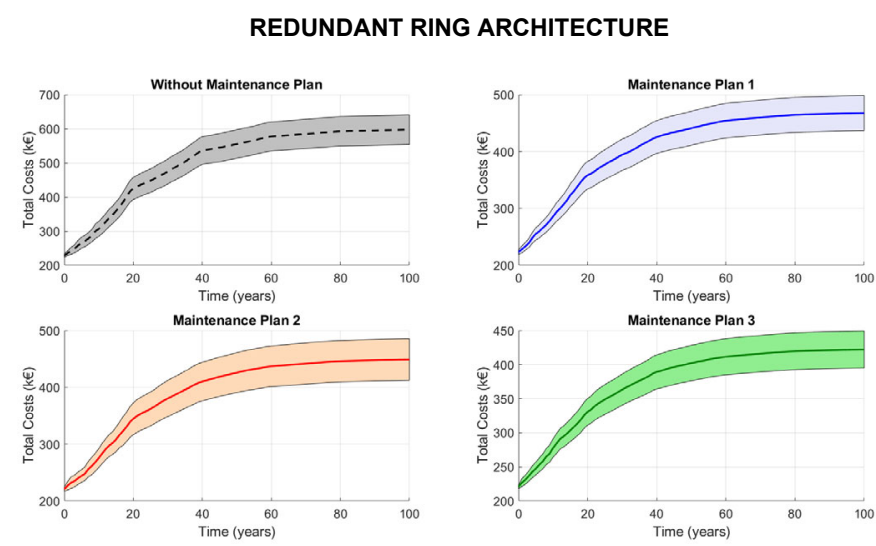

Fig. 15. Total discounted costs of the substation for the redundant ring architecture [7].

For all maintenance plans and communication architectures considered, the curve of the mean value and variance of the total discounted costs seems to converge to a certain value. This happens due to the monetary value of time, and so the amount to be paid relative to the present decreases throughout the lifetime of the substation.

Once again, it is possible to see an improvement in the total discounted costs, as these decrease with each maintenance plan. Furthermore, there is an increase in variance over time, which is expected since the uncertainty in the operational behavior of the substation increases in the same way. However, the most interesting result of this analysis is that there is no reduction in the total discounted costs as the redundancy of communication architectures increases, since it is the star architecture with the best results in economic terms.

In Table 5 and

Table 6, a summary of the results obtained for all architectures is presented. The variance values shown for both availability and total discounted cost correspond to the maximum obtained from the results.

Table 5. Average availability for each configuration [7].

\begin{tabular}{lcccc} 
& \multicolumn{4}{c}{ Availability (\%) } \\
\cline { 2 - 5 } Configuration & No Plan & Plan 1 & Plan 2 & Plan 3 \\
\hline Cascade & 99.7929 & 99.8320 & 99.8408 & 99.8474 \\
& $( \pm 1.5 \%)$ & $( \pm 0.7 \%)$ & $( \pm 0.6 \%)$ & $( \pm 0.5 \%)$ \\
Ring & 99.8294 & 99.8638 & 99.8733 & 99.8838 \\
& $( \pm 1.2 \%)$ & $( \pm 0.4 \%)$ & $( \pm 0.4 \%)$ & $( \pm 0.2 \%)$ \\
Star & 99.8313 & 99.8640 & 99.8756 & 99.8849 \\
& $( \pm 1.2 \%)$ & $( \pm 0.6 \%)$ & $( \pm 0.3 \%)$ & $( \pm 0.2 \%)$ \\
Redundant & 99.8477 & 99.8803 & 99.8849 & 99.8979 \\
Ring & $( \pm 0.9 \%)$ & $( \pm 0.4 \%)$ & $( \pm 0.3 \%)$ & $( \pm 0.2 \%)$ \\
\hline
\end{tabular}

Table 6. Total discounted costs for each configuration [7].

\begin{tabular}{lcccc}
\cline { 2 - 5 } Configuration & No Plan & Plan 1 & Plan 2 & Plan 3 \\
\hline \multirow{2}{*}{ Cascade } & 809.09 & 664.95 & 638.68 & 618.35 \\
& $( \pm 7.2 \%)$ & $( \pm 2.9 \%)$ & $( \pm 4.7 \%)$ & $( \pm 8.1 \%)$ \\
\multirow{2}{*}{ Ring } & 574.14 & 472.63 & 455.68 & 414.68 \\
& $( \pm 5.9 \%)$ & $( \pm 5.5 \%)$ & $( \pm 5.6 \%)$ & $( \pm 5.1 \%)$ \\
Star & 586.85 & 469.99 & 438.78 & 403.39 \\
& $( \pm 7.9 \%)$ & $( \pm 6.2 \%)$ & $( \pm 6.6 \%)$ & $( \pm 5.9 \%)$ \\
Redundant & 596.99 & 467.57 & 448.85 & 422.04 \\
Ring & $( \pm 7.3 \%)$ & $( \pm 6.6 \%)$ & $( \pm 8.2 \%)$ & $( \pm 6.4 \%)$ \\
\hline
\end{tabular}


In the case of a real system in operation, it is not expectable that the failure rate of a certain equipment remains exactly the same throughout its lifetime, even if it is maintained regularly. Moreover, the field of reliability physics deals with the subject of planned obsolescence of equipment. This term describes a strategy, adopted by many companies nowadays, which consists of deliberately ensuring that the current version of a given component will become out of date or even inoperable within a certain amount of time. This guarantees the need for consumers to seek replacements in the future, thus increasing demand. Therefore, an alternative analysis considering equipment degradation is performed. The purpose of this analysis is to defy the useful life period of the bathtub curve from Fig. 2 and develop a new model as close to reality as possible.

In this case, the useful life period is extended. Thus, the constant failure rate model cannot be used. A failure rate degradation function, $\lambda_{d}(t)$, was then developed to model the behavior of the new useful life period, which can be analyzed in equation (12). This function considers the original failure rate, $\lambda_{0}$, and the lifetime, $L T$, of the component in the substation, which in this work is 20 years for all components.

$$
\lambda_{d}(t)=\lambda_{0}+e^{-(t-L T)}
$$

Since the failure rate is not constant, the reliability of each component cannot be computed the same way. Therefore, from equation (3), the reliability of each component considering its degradation, $R_{d}(t)$, can be computed through equation (13).

$$
R_{d}(t)=e^{-\left(\lambda_{0} \cdot t+e^{-(t-L T)}\right)}
$$

Similar to what was presented in sub-section 5.1, the results of this proposal were analyzed in the form of graphs considering all preventive maintenance plans and communication architectures. As expected, there is a general worsening in the results, as reliability and availability values decrease while total discounted costs increase for all scenarios. Nevertheless, the behavior of the results obtained in this proposal are similar to the ones observed when applying the constant failure rate model, as shown in Fig. 16 to Fig. 21 and presented in

Table 7 and Table 8.

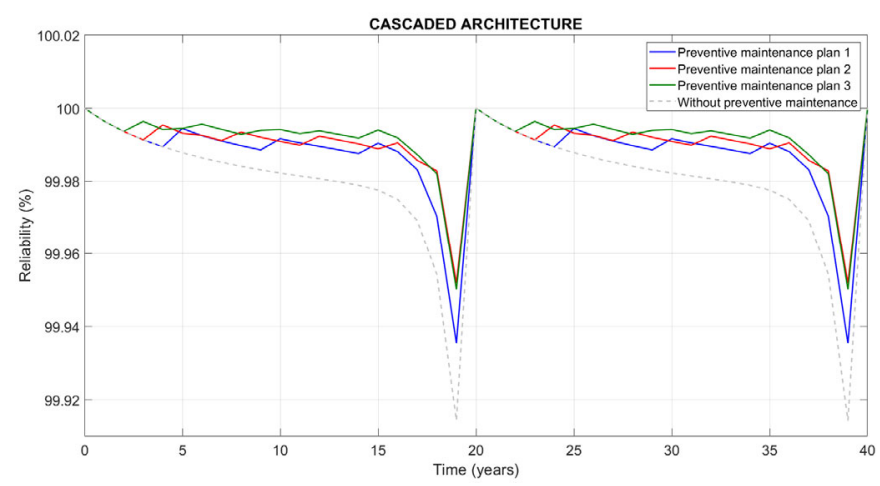

Fig. 16. Reliability curves for the cascaded architecture considering equipment degradation [7].

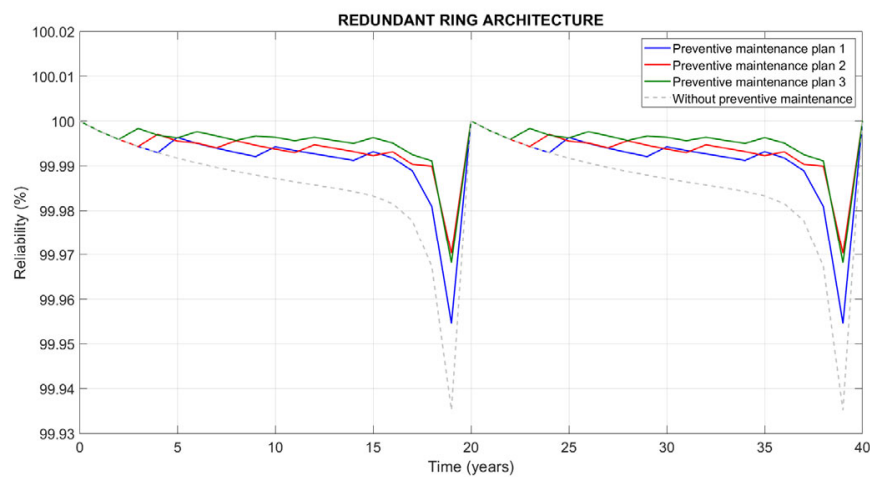

Fig. 17. Reliability curves for the redundant ring architecture considering equipment degradation [7]. 

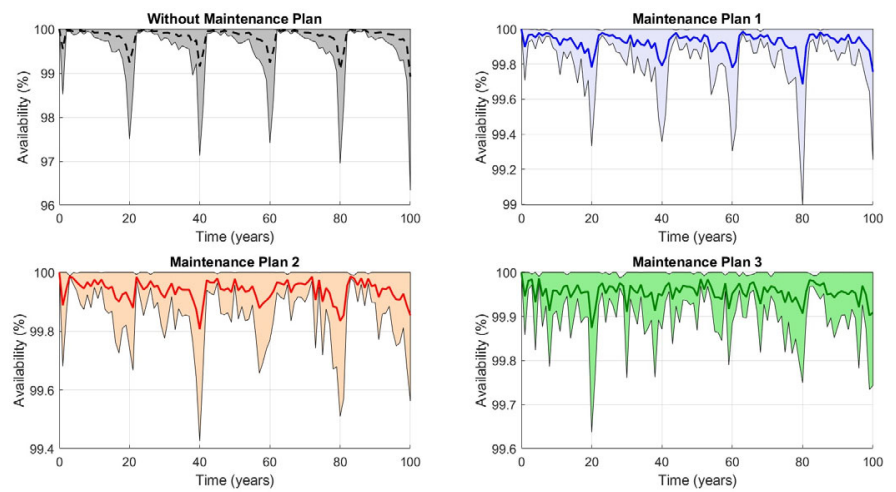

Fig. 18. Availability of the substation for the cascaded architecture considering equipment degradation [7].
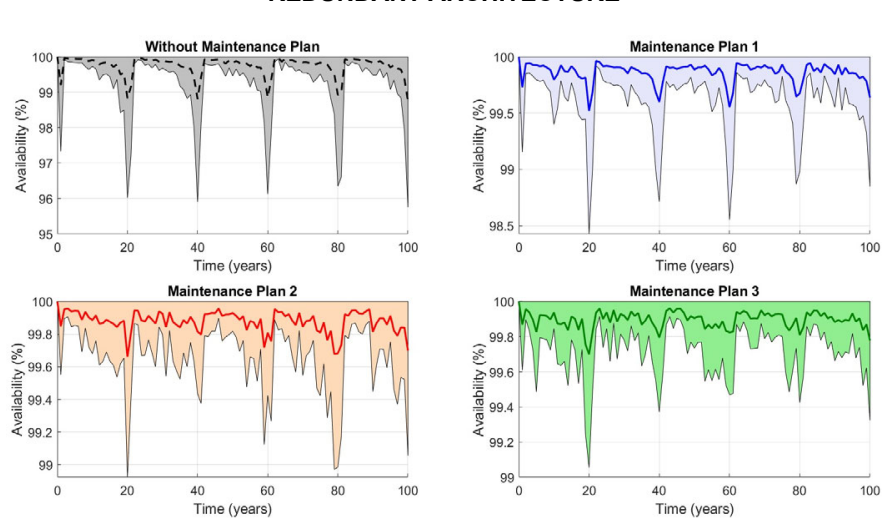

Fig. 19. Availability of the substation for the redundant ring architecture considering equipment degradation [7].

CASCADED ARCHITECTURE
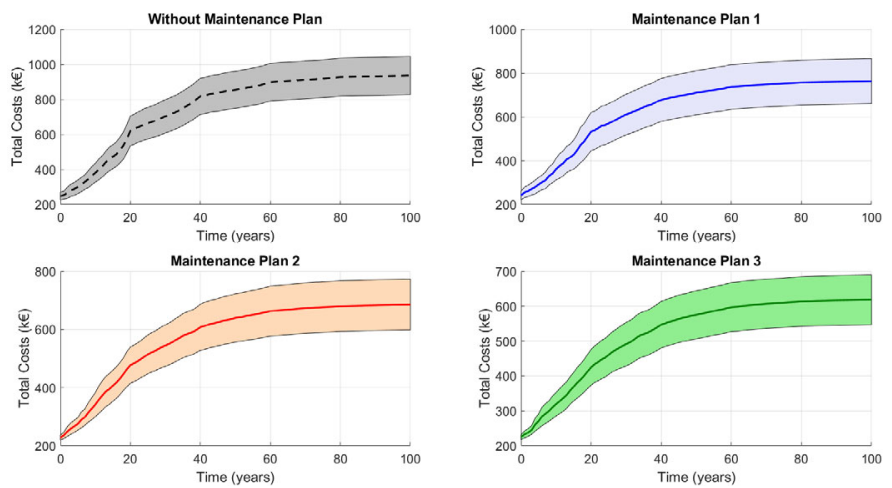

Fig. 20. Total discounted costs of the substation for the cascaded architecture considering equipment degradation [7]. 

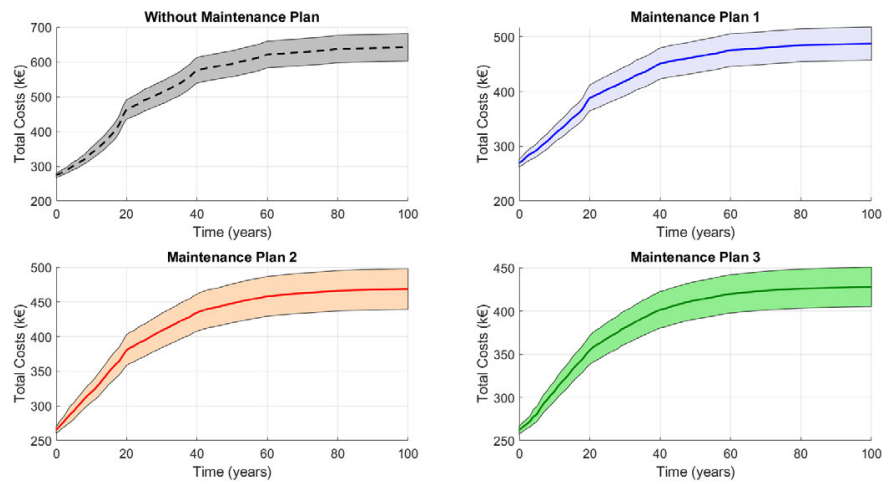

Fig. 21. Total discounted costs of the substation for the redundant ring architecture considering equipment degradation [7].

Table 7. Average availability considering equipment degradation [7].

\begin{tabular}{lcccc} 
& \multicolumn{4}{c}{ Availability (\%) } \\
\hline Configuration & No Plan & Plan 1 & Plan 2 & Plan 3 \\
\hline \multirow{2}{*}{ Cascade } & 99.7714 & 99.8170 & 99.8347 & 99.8462 \\
& $( \pm 1.9 \%)$ & $( \pm 0.9 \%)$ & $( \pm 0.8 \%)$ & $( \pm 0.7 \%)$ \\
Ring & 99.8082 & 99.8574 & 99.8664 & 99.8764 \\
& $( \pm 1.6 \%)$ & $( \pm 0.5 \%)$ & $( \pm 0.5 \%)$ & $( \pm 0.3 \%)$ \\
Star & 99.8064 & 99.8506 & 99.8692 & 99.8817 \\
Redundant & $( \pm 1.6 \%)$ & $( \pm 0.8 \%)$ & $( \pm 0.4 \%)$ & $( \pm 0.3 \%)$ \\
Ring & 99.8210 & 99.8699 & 99.8815 & 99.8962 \\
& $( \pm 1.2 \%)$ & $( \pm 0.5 \%)$ & $( \pm 0.4 \%)$ & $( \pm 0.3 \%)$ \\
\hline
\end{tabular}

Table 8. Total discounted costs considering equipment degradation [7].

\begin{tabular}{lcccc}
\cline { 2 - 5 } Configuration & \multicolumn{4}{c}{ Notal Discounted Costs $(\mathrm{k} €)$} \\
\hline \multirow{2}{*}{ Cascade } & 922.17 & 714.96 & 654.47 & 598.50 \\
& $( \pm 7.2 \%)$ & $( \pm 2.9 \%)$ & $( \pm 4.7 \%)$ & $( \pm 8.1 \%)$ \\
\multirow{2}{*}{ Ring } & 671.42 & 509.88 & 472.30 & 441.24 \\
& $( \pm 5.9 \%)$ & $( \pm 5.5 \%)$ & $( \pm 5.6 \%)$ & $( \pm 5.1 \%)$ \\
Star & 668.58 & 495.49 & 474.78 & 433.09 \\
\multirow{2}{*}{ Redundant } & $( \pm 7.9 \%)$ & $( \pm 6.2 \%)$ & $( \pm 6.6 \%)$ & $( \pm 5.9 \%)$ \\
Ring & $( \pm 2.14$ & 487.50 & 468.72 & 428.16 \\
& $( \pm 7.3 \%)$ & $( \pm 6.6 \%)$ & $( \pm 8.2 \%)$ & $( \pm 6.4 \%)$ \\
\hline
\end{tabular}

Results show that the impact of the proposed approach is significant both in operational and economical terms. The decrease in availability relatively to the scenario without equipment degradation is evident, which is translated in a considerable increase in the total discounted costs of the substation. However, in this case the architecture which presents the best trade-off between availability and total discounted costs is the redundant ring architecture, with the application of preventive maintenance plan 3 . Since the availability reduces, the penalties for energy not supplied increase significantly, which is determinant for the difference between the two models.

\section{Conclusion}

From the results presented, one can conclude that, independently on the communication architecture implemented in the substation, a more detailed preventive maintenance plan reveals to be the best option both in an operational and economical perspective. 
Although the impact of failures of SAS components in the operation of the substation is residual, investment in redundant communication architectures proves to be beneficial in an operational perspective. The same, however, may not be true from an economic point of view.

Thus, the main contribution of this work is the proposal of a method which allows the identification of opportunities for companies in the electricity sector to involve themselves in the design and construction of digital substations, working towards a safer, reliable and economically efficient operation. Moreover, the use of the Markov-Monte Carlo simulation method reveals to be a good way for the reliability and availability analysis of any system.

\section{Acknowledgements}

This work was supported by national funds through the Fundação para a Ciência e a Tecnologia, I.P., through IDMEC, under LAETA, Project UID/EMS/50022/2020, and by Secretaría Nacional de Educación Superior, Ciencia, Tecnología e Innovación (SENESCYT) of the Ecuadorian Government through fellowship CZ05-0002912017.

This paper is an extract from the academic research conducted by the first author as requirement to obtain the Master of Science Degree in Electrical and Computer Engineering at Instituto Superior Técnico, Universidade de Lisboa, under the supervision of Prof. Paulo J. Costa Branco and the co-supervision of Eng. Andrés A. Zúñiga.

\section{References}

[1] N. Liu, M. Panteli, P.A. Crossley, Reliability evaluation of a substation automation system communication network based on IEC 61850, in: 12th IET International Conference on Developments in Power System Protection (DPSP 2014), 2014: pp. 1-6. https://doi.org/10.1049/cp.2014.0057.

[2] R. Billinton, W. Li, Reliability Assessment of Electric Power Systems Using Monte Carlo Methods, Springer Science+Business Media, New York, 1994. https://doi.org/10.1007/978-1-4899-1346-3.

[3] U.B. Anombem, Evaluation of IEC 61850 Process Bus Architecture and Reliability, The University of Manchester, 2012.

[4] ALSTOM, Network Protection \& Automation Guide, Alstom Grid, 2011.

[5] J. Soh, What is Scaling, and Why Is It Necessary?, (n.d.). https://blog.phoenixcontact.com/marketingsea/2016/03/what-is-iec-61850-and-why-is-it-necessary/ (accessed November 10, 2020).

[6] T.S. Sidhu, M.G. Kanabar, S. Member, P. Palak, A. International, E.C. Iec, Implementation Issues with IEC 61850 Based Substation Automation Systems, in: Fifteenth National Power Systems Conference (NPSC), Bombay, 2008: pp. 473-478.

[7] J.P. de S. Tarquínio, Reliability Analysis of the Control and Automation System in Electrical Substations, Universidade de Lisboa, 2021.

[8] M. Rausand, A. Høyland, System Reliability Theory, Second Edi, John Wiley \& Sons, Inc., Hoboken, New Jersey, 2004.

[9] IEEE PSRC H6 Special Report, Application Considerations of IEC 61850 / UCA 2 for Substation Ethernet Local Area Network Communication for Protection and Control, 2005. https://www.pespsrc.org/kb/published/reports/H6Paper-App Consider of IEC61850\&UCA_072205_083105.pdf.

[10] M.G. Kanabar, T.S. Sidhu, Reliability and availability analysis of IEC 61850 based substation communication architectures, in: 2009 IEEE Power and Energy Society General Meeting, PES '09, 2009: pp. 1-8. https://doi.org/10.1109/PES.2009.5276001.

[11] A.N.S. Baleia, Failure Modes and Effects Analysis (FMEA) for Smart Electrical Distribution Systems, Universidade de Lisboa, 2018.

[12] A. Klein, Hard Drive Stats for Q3 2017, (n.d.). https://www.backblaze.com/blog/hard-drive-failure-ratesq3-2017 (accessed July 30, 2021).

[13] EKE Electronics, Human Machine Interface (HMI), (n.d.). https://www.eke-electronics.com/humanmachine-interface (accessed July 30, 2021).

[14] CISCO, Cisco Industrial Ethernet 2000 Series Switches - Data Sheet, (2016) 1-12. http://www.cisco.com/c/en/us/products/switches/industrial-ethernet-2000-series- 
switches/index.html?CAMPAIGN=ie2000\&COUNTRY_SITE=us\&POSITION=vanity\&REFERRING_SI TE=vanity\&CREATIVE=go_ie 2000 .

[15] F. Berghmans, S. Eve, M. Held, An Introduction To Reliability of Optical Components and Fiber Optics Sensors, in: W.J. Bock, I. Gannot, S. Tanev (Eds.), Optical Waveguide Sensing and Imaging. NATO Science for Peace and Security Series B: Physics and Biophysics, Springer Science+Business Media, B.V., Dordrecht, 2008: pp. 73-100. https://doi.org/10.1007/978-1-4020-6952-9.

[16] J. Bettler, J. Silva, D. Morman, R. Abboud, D. Bowen, E. Cenzon, D. Dolezilek, J. Silva, S.C. Edison, R. Abboud, D. Bowen, E. Cenzon, D. Dolezilek, S.E. Laboratories, Case Studies of IEC 61850 Process Bus Systems Using GOOSE and Sampled Values : Recent Installations and Research, in: 74th Annual Georgia Tech Protective Relaying Conference, 2021: pp. 1-12.

[17] V. Letloff, Convener, M. Eckl, P. Jenaker, M. Herzig, F. Koers, S.A. Kunsman, I. Lorencin, R.S.J. Loken, A.L. de Viñaspre, M. Loukkalahati, I.P. de Siqueira, M. Paulino, M. Petrini, J. Robertson, V. Tiesmäki, P. Wittlinger, Jo. Wright, Maintenance Strategies for Digital Substation Automation Systems, ELECTRA. (2011) 67-71. 